\title{
Impacto de un programa comunitario para la malnutrición infantil
}

\author{
Impact of a community program for child malnutrition \\ Clara Denisse Perdomo ${ }^{a}$, Elizabeth Raquel Rodríguez ${ }^{\mathrm{b}}$, Héctor Carrasco Magallanes $^{c}$, \\ Hugo Ernesto Flores Navarro ${ }^{\mathrm{d}}$, Saira Elvira Matul Pérez ${ }^{\mathrm{e}}$, Daniela Moyano ${ }^{\mathrm{f}}$
}

\author{
aLicenciada en Nutrición. Universidad Nacional de Formosa. Consejo Nacional de Investigaciones Científicas y Técnicas, CONICET \\ bicenciada en Nutrición. Escuela de Nutrición, Facultad de Ciencias Médicas, Universidad Nacional de Córdoba \\ 'Medico, Magister en Salud Pública. Compañeros en Salud (filial Mexicana de Partners in Health). Harvard T.H Chan, School of Public Health \\ dMedico. Compañeros en Salud (filial Mexicana de Partners in Health) \\ eLicenciada en Enfermería. Secretaria de Salud de México \\ fLicenciada en Nutrición. Magíster en Salud Pública. Escuela de Nutrición, Facultad de Ciencias Médicas, Universidad Nacional de Córdoba
}

Recibido: 8 de octubre de 2018; Aceptado: 1 de abril de 2019

\begin{abstract}
Resumen
Objetivo: Evaluar el impacto de un programa comunitario destinado a mejorar la malnutrición de niños y niñas de una comunidad rural del Estado de Chiapas, México, 2013. Material y Método: Estudio descriptivo de la evaluación de un programa a partir de una base de datos secundaria con datos nutricionales en 113 niños menores de cinco años de una zona rural de México. La intervención y el relevamiento se realizaron durante el 2013. Se registraron mediciones basales y a los 4 meses. Para el cálculo de indicadores del estado nutricional se utilizó el Software Anthro de la Organización Mundial de la Salud (OMS). Se estimaron: Peso para edad (P/E); Talla para edad (T/E); Peso para talla (P/T); Índice de masa corporal para la edad (IMC/E), según los lineamientos de la OMS. Se calcularon medidas de posición y dispersión, prueba T de Student, Kruskal-Wallis, test de MacNemar para datos pareados y regresión lineal simple. Resultados: Entre el inicio y final la mediana del Z Peso/ talla pasó de -0,7 (p25-1,24; p75 -0,01) a -0,62 (p25 -1,09; p75 -0,15). La prevalencia de bajo peso descendió de 5,31\% (IC 2,38-11,44) a 4,42\% (IC 1,83-10,32) (Z Score IMC/edad). El peso adecuado según Z Score Peso/talla aumentó de 78,76\% (IC 70,12-85,43) a 84,96\% (76,98-90,51). En el subgrupo con bajo peso inicial la media de Z IMC/edad y Z Peso/talla aumentó $0,4(\mathrm{p}=0,003)$. El cambio en la media de $Z$ Peso/talla fue de 0,02 puntos en el subgrupo que recibió programa de trasferencia directa y de $-0,3$ en el que no $(p=0,020)$. Conclusiones: Se concluye que el programa comunitario durante los 4 meses de implementación contribuyó a mejorar algunos indicadores antropométricos; aunque no se encontraron efectos aparentes en indicadores relacionados a la desnutrición crónica.
\end{abstract}

Palabras clave:

Niño;

Servicios de salud comunitaria; Desnutrición; Programas y Políticas de Nutrición y Alimentación 


\section{Abstract}

Objective: To evaluate the impact of a community program aimed at improving the children malnutrition in a rural community of the State of Chiapas, Mexico, 2013. Material and Method: Descriptive study of the evaluation program from a secondary database of nutritional data registry of 113 children under five years of age in a rural area of Mexico. The intervention and the survey were carried out during 2013. Baseline and 4-month measurements were recorded. The World Health Organization (WHO) Anthro software was used to calculate nutritional status indicators. According to WHO guidelines, the following data were estimated: weight for age (W/A), height for age (H/A), weight for height $(\mathrm{W} / \mathrm{H})$, and Body mass index for age (BMI/A). Position and dispersion measures were calculated; Student's T-test, Kruskal-Wallis, and MacNemar test were used for paired data and linear regression. Results: Between the beginning and the end, the median of the $\mathrm{Z} \mathrm{W} / \mathrm{H}$ went from -0.7 (p25 -1.24, p75 -0.01) to -0.62 (p25-1.09, p75 -0.15). The prevalence of low weight decreased from $5.31 \%$ (CI 2.38-11.44) to $4.42 \%$ (CI 1.83-10.32) (Z BMI/A). The appropriate weight according to $\mathrm{Z}$ score $\mathrm{W} / \mathrm{H}$ increased from $78.76 \%$ (CI 70.12-85.43) to $84.96 \%$ (76.98-90.51). In the subgroup with low initial weight, the mean of $\mathrm{Z} \mathrm{BMI} / \mathrm{A}$ and $\mathrm{ZW} / \mathrm{H}$ increased $0.4(\mathrm{p}=0.003)$. The change in the mean of $\mathrm{ZW} / \mathrm{H}$ was 0.02 points in the subgroup that received the direct transfer program and of -0.3 in which it did not $(p=0.020)$. Conclusions: It is concluded that the community program during the four months of implementation contributed to improve some anthropometric indicators, although no apparent effects were found in indicators related to chronic malnutrition.

\section{Keywords:}

Children;

Community Health

Services;

Malnutrition;

Nutrition Programs

and Policies

\section{Introducción}

En las últimas décadas en América Latina se han observado mejoras en algunos aspectos relacionados a la protección de los derechos de niños y niñas ${ }^{1} \sin$ embargo, la evidencia pone en relieve diversas problemáticas que afectan negativamente su salud y que repercuten en el desarrollo sano y armónico ${ }^{2,3}$.

En México en el año 2014 se observó que el 55,2\% de niños y niñas entre 2 y 5 años se encontraron bajo la línea de pobreza y un $13,1 \%$ presentó pobreza extrema ${ }^{3}$, siendo uno de los principales determinantes sociales de la salud y nutrición ${ }^{4}$. En particular la desnutrición, durante los primeros años de vida es causa y producto de la pobreza ${ }^{5}$.

La malnutrición infantil es un grave problema en América Latina y el Caribe que viola el derecho a la vida y constituye un estado patológico multicausal y con efectos importantes en el desarrollo del niño $0^{6}$. Incluye procesos biológicos socialmente determinados ${ }^{7,8}$ estando relacionada a las condiciones sociales y ambientales?.

La malnutrición abarca la desnutrición (emaciación, retraso del crecimiento e insuficiencia ponderal), los desequilibrios de vitaminas o minerales, el sobrepeso, la obesidad, y las enfermedades no transmisibles relacionadas con la alimentación ${ }^{10}$.

Según datos de la Organización Mundial de la Salud (OMS) 52 millones de niños menores de 5 años presentan emaciación, 17 millones emaciación grave y 155 millones retraso del crecimiento, mientras que 41 millones presentan sobrepeso u obesidad ${ }^{10}$.
En México, a partir de la Encuesta Nacional de Salud y Nutrición de Medio Camino 2016 se estimó que la prevalencia de sobrepeso fue de 17,9\% y de obesidad de $15,3 \%$ en niños/as entre 5 a 11 años ${ }^{11}$. Sin embargo, la desnutrición tanto aguda como crónica sigue siendo un problema importante en el país ${ }^{12-14}$, donde se observó que durante el periodo de 1990 a 2009 han ocurrido cerca de 35 mil muertes de niños menores de cinco años a causa de la desnutrición ${ }^{12}$.

Como lo platean algunos autores, la malnutrición en sus múltiples formas se constituyen actualmente en un desafío para la salud pública ${ }^{15,16}$ teniendo importantes impactos en las esferas física, psicológica y social ${ }^{17}$ de niños y niñas.

Existen importantes brechas en el estado nutricional de los niños provenientes de zonas urbanas con respecto a los de zonas rurales. En México la prevalencia de desnutrición crónica en la población rural es el doble que en la de zona urbana ${ }^{14}$.

Una revisión sistemática publicada recientemente, puso en evidencia que los factores asociados con el retraso en el crecimiento y el bajo peso infantil podrían derivarse de la esfera social y ambiental, entre los que se encuentran la baja educación e inadecuado estado de nutrición y salud de la madre, pobreza del hogar y la residencia rural ${ }^{18}$.

Otro estudio comprobó que el retraso en el crecimiento en niños y niñas se asoció con una menor escolarización, un menor rendimiento escolar y un aumento de la probabilidad de vivir en la pobreza durante la vida adulta ${ }^{19}$, por este motivo la malnutrición infantil es un problema prioritario en la agenda de salud pú- 
blica que requerirá de intervenciones complejas a nivel de las comunidades con un enfoque holístico y participativo $^{18,20,21}$.

Este estudio surge en el marco de un programa comunitario llevado a cabo por la organización internacional sin fines de lucro Partners in Health (PIH) y tuvo como objetivo principal evaluar el impacto de un programa comunitario destinado a mejorar la malnutrición de niños y niñas de una comunidad rural del Estado de Chiapas, México, 2013.

\section{Material y Método}

\section{Tipo de estudio}

Estudio descriptivo, analítico y longitudinal de evaluación de un programa a partir de una base de datos secundaria proveniente del registro de datos nutricionales individuales de niños/as menores de cinco años perteneciente a la organización internacional Partners in Health o Compañeros en Salud (en sus siglas en español) en la comunidad rural de La Soledad del Estado de Chiapas, México, durante el año 2013.

Compañeros en Salud trabaja activamente en Sierra Madre de Chiapas, el estado más pobre de México, en convenio con la Secretaría de Salud. Datos provenientes de la organización indicaron que en el 2016 se brindaron más de 28.000 consultas médicas a la población, 142 comunidades se encuentran bajo cobertura y más de 300 pacientes fueron acompañados para recibir atención especializada ${ }^{22}$.

\section{Participantes}

Los criterios de inclusión durante el programa fueron el presentar por lo menos uno de los siguientes diagnósticos al inicio del programa: desnutrición aguda (bajo peso medidos por el Z IMC/edad o Z Peso/ talla), desnutrición crónica (muy baja talla; baja talla medido por el $\mathrm{Z}$ Talla/edad), y/o tener un peso normal, pero estar en riesgo de desnutrición aguda o crónica (definido por los siguientes criterios: diagnóstico de alerta de bajo peso/edad, alerta de baja talla/edad o alerta de bajo peso/talla). Además, pertenecer a la comunidad bajo cobertura del programa, cercanía de la clínica de salud, tener hasta cinco años y presentar autorización por escrito o verbal de la madre/padre o tutor.

Dado que fue un programa comunitario no se aplicó un tamaño muestral y se incluyeron al grupo de intervención (GI) a todos los niños de ambos sexos que cumplían con los criterios de inclusión al programa.

Para la recolección de datos sobre diagnóstico del estado nutricional y datos sociodemográficos del niño/a se implementó un instrumento de recolección que consistió en una guía de observación y registro de mediciones antropométricas que fue administrado por el personal de la clínica salud de la comunidad de La Soledad.

Previo a la implementación del programa, el equipo de salud de la clínica recibió capacitación sobre técnicas de mediciones antropométricas siguiendo los lineamientos del manual de capacitación para personal de salud "Vigilancia de la Nutrición y Crecimiento del Niño" de la Secretaría de Salud de México con la finalidad de estandarizar las mediciones. Para estimar el peso se utilizó una báscula pesa/bebé en los menores de 2 años y una báscula de plataforma para mayores de 2 años con precisión de $100 \mathrm{~g}$ y para la talla por medio de un infantómetro paro menores de 2 años y estadímetro paro niños mayores de 2 años.

Durante el relevamiento de datos al inicio del programa se preguntó a la madre (autoreporte) si su niño/a fue nacido a término para corregir la edad en los casos de niños prematuros.

En este análisis, para el cálculo de indicadores del estado nutricional de niños/as se utilizó el Software Anthro de la Organización Mundial de la Salud (OMS). Se estimaron: Peso para la edad (P/E); Talla para la edad (T/E); Peso para la talla $(\mathrm{P} / \mathrm{T})$; Índice de masa corporal para la edad (IMC/E) según los lineamientos de la $\mathrm{OMS}^{23}$.

\section{Actividades llevadas a cabo desde el programa}

Antes de empezar el programa se invitaron a participar a las madres de niños con criterios de inclusión captados a través de consulta espontánea, por derivación del médico o enfermera de la clínica de salud, mediante anuncios (con megáfonos), y/o visitas a los hogares.

El periodo que se tomó para realizar este análisis fue de 4 meses (mediciones basales y a los 4 meses) ya que es el tiempo que se implementó el programa comunitario y del que se disponen de datos registrados.

La metodología de planificación y ejecución de las actividades del programa se basó en los niveles de abordaje del Modelo Ecológico ${ }^{24}$ que tuvo como eje principal al niño considerando la interrelación de elementos individuales, familiares, sociales y comunitarios.

\section{Microsistema}

Relacionado al ambiente más cercano de niño con malnutrición, es el lugar en el que la persona puede interactuar cara a cara fácilmente, como en el hogar y el sistema de salud.

\section{*Sistema de salud}

- Asesoría, educación y screening del estado nutricional por parte de profesionales de la salud en consultorio externo.

- Consultas mensuales médicas y nutricionales in- 
dividuales de seguimiento con implementación de recordatorio alimentario 24 horas, asesoría nutricional personalizada, implementación de la encuesta de factores de riesgo de desnutrición ad hoc, evaluación y seguimiento del estado nutricional e implementación de la ELCSA (Escala Latinoamericana y Caribeña de Seguridad Alimentaria).

- Suplementación nutricional (multivitamínicos, hierro) y probiótico con lactobacillus rhamnosus GG (para el manejo de diarreas).

- Control e implementación del calendario de desparasitación.

- Medición bioquímicas de hemoglobina.

- Asesoría e instancias de sensibilización a madres y cuidadoras en la sala de espera de la clínica de salud.

\footnotetext{
${ }^{*}$ Hogar

- Visitas domiciliaras mensuales planificadas a niños con mayor riesgo social.
}

\section{Mesosistema}

Comprende las interrelaciones de dos o más entornos en los que el niño en desarrollo participa activamente.

- Cocina comunitaria a cargo de las madres: con eje principal en la organización y empoderamiento de madres para llevar a cabo actividades de recaudación de fondos, compra y elaboración de alimentos.

\section{Exosistema}

Se refiere a uno o más entornos que no incluyen al niño en desarrollo como participante activo, pero en los cuales se producen los hechos que lo afecta.

- Talleres comunitarios de educación: modalidad de talleres mensuales teórico-prácticos planificados y ejecutados por profesionales de la salud. Las temáticas fueron sobre alimentación saludable, alimentación y cultura, cocina saludable, e higiene. Se contó con herramientas y materiales didácticos.

- Micro-emprendimientos mediante obtención de micro-créditos destinados a mujeres: a través de un programa gubernamental.

- Obtención de animales de granja para autoconsumo: selección de familias, capacitación y entrega de animales de granja con posterior seguimiento.

- Elaboración de incubadora de huevos casera: diseño y elaboración de una incubadora de huevos casera.

- Capacitación e implementación de huertas comunitarias: en espacios comunitarios con el objetivo de constituirse en una herramienta socio-pedagógica y de sensibilización para progresar hacia la huerta familiar.

\section{Macrosistema}

Lo configuran la cultura y la subcultura en la que se desenvuelve la persona y todos los individuos de su sociedad. Es el contexto más amplio y remite a las formas de organización social.

- Acuerdos intersectoriales: con el área de educación a nivel local, líderes comunitarios, organismos gubernamentales y no gubernamentales. Se articuló actividades con los programas Diconsa (asistencia alimentaria) y Oportunidades (transferencia directa).

\section{Variables dependientes}

- Puntuación Z Talla/edad, Z IMC/edad y Z Peso/ talla.

- Estado nutricional según Z Score IMC/edad (Bajo peso; Alerta de Bajo peso, Peso adecuado; Alto peso y Muy alto peso).

- Estado nutricional según Z Score Peso/talla (Desnutrición aguda severa; Desnutrición aguda moderada; Riesgo de desnutrición aguda; Peso adecuado para la talla; Riesgo de sobrepeso o sobrepeso; Obesidad).

- Estado nutricional según Z Score Talla/edad (Muy baja talla; Baja talla; Alerta de Baja talla; Talla adecuada y Alta talla).

\section{Variables independientes}

- Programa de protección social: definido como auto-reporte al momento de ingresar a la intervención de estar recibiendo protección social por medio del programa de asistencia alimentaria Diconsa y/o transferencia directa de dinero por medio del Programa Oportunidades.

- Edad del niño/a (en meses cumplidos).

- Sexo del niño/a (varón/mujer).

\section{Análisis estadístico}

Se realizó un análisis descriptivo de las variables de interés utilizando medidas de posición y dispersión (media, mediana, error estándar y percentilos 25 y 75) de acuerdo con la distribución de los datos en el caso de las variables continuas; así como frecuencia absoluta y relativa para los datos categóricos.

Se estimaron prevalencias de diagnóstico de estado nutricional y su intervalo de confianza del $95 \%$.

Se evaluó la normalidad de la distribución de los datos continuos (peso corporal, talla y puntuación Z) por medio de métodos gráficos (histograma), la prueba de Shapiro-Wilk y para el análisis de las varianzas se utilizó la prueba de Bartlett.

Se eliminaron de la base de datos sujetos que no tuvieron datos basales en todas las variables bajo análisis. El cálculo de las puntuaciones $\mathrm{Z}$ y de los diagnósticos de estado nutricional se realizaron siguiendo los linea- 
mientos de la OMS realizados por dos investigadores independientes. En todos los casos se utilizaron datos estandarizados a través de la puntuación Z.

Los análisis de cambios en el estado nutricional al inicio y final del programa a nivel individual se hicieron a través de prueba T de Student, la no paramétrica de Kruskal-Wallis, el test de MacNemar para datos pareados y regresión lineal simple. La significancia estadística en todos los casos fue de de $\mathrm{p}<0,05$ y nivel de confianza del $95 \%$. El software estadístico utilizado para todos los análisis fue Stata 14.

\section{Aspectos éticos}

El presente estudio se llevó a cabo a partir del análisis de una base de datos secundaria de datos elaborada por la organización Compañeros en Salud durante su trabajo en territorio en el marco de un programa implementado durante el año 2013.

Al comienzo del programa se solicitó a las madres, padres y/o tutores de niños y niñas un consentimiento por escrito y/o verbal para la participación de su hijo/a.

El equipo de investigación solicitó por escrito un permiso para el uso de la base y antes de ser analizada estuvo previamente de-identificada por el organismo responsable.

El protocolo fue evaluado y aprobado por un comité de ética y se encuentra exento de firma de consentimiento informado ya que se trabajó con datos secundarios de un registro de salud pública.

\section{Resultados}

La muestra al inicio del programa fue de 113 niños/ as representada por un $46 \%$ de sexo masculino y un $54 \%$ de femenino y al final del programa fue de 112 sujetos (tasa de pérdida del $1 \%$ ).

La media de edad en la etapa basal fue de 31 meses (IC 27,9-34,0), siendo inferior en los niños (media de 28,2 meses con un IC $24,0-32,4$ ) que en las niñas (media de 33,3 meses con IC 29,0-37,7).

El peso corporal inicial fue levemente superior en las niñas con respecto a los niños (10,9 Kg vs. 10,6 Kg), donde la talla siguió esta misma tendencia $(85,1 \mathrm{~cm}$ vs. $82,5 \mathrm{~cm})$. Un $62 \%$ de la muestra estaba recibiendo programa de transferencia directa de dinero y un $33,6 \%$ asistencia alimentaria (tabla 1).

En la tabla 2 se presenta la comparación de los indicadores antropométricos al incio y al final del programa comunitario. En los indicadores de Z IMC/edad y Talla/edad la mediana se mantuvo similar en ambas etapas, mientras que la mediana del $\mathrm{Z}$ Peso/talla pasó de -0,7 (p25-1,2; p75 -0,0) a -0,6 (p25 -1,1; p75 -0,1).

El diagnóstico nutricional estimado por el Z Score IMC/edad mostró que la prevalencia de bajo peso pasó de 5,3\% (IC 2,4-11,4) a 4,42 \% (IC 1,8-10,3) entre el inicio y final del programa, donde la categoría de peso adecuado fue de $83,2 \%$ (IC 75,9-89,1) a 86,7 (IC 79,091,9) entre dichas etapas (Tabla 2).

En cuanto al Z Score Talla/edad, se observó que la categoría diagnóstica de muy baja talla pasó de 10,6\% (IC 6,1-17,9) a 6,2\% (IC 2,9-12,5), mientras que la categoría de baja talla se mantuvo sin cambios en una prevalencia del 30,1\% (IC 22,2-39,3) entre el inicio y el final de la intervención (Tabla 2).

Cuando se analizó el estado nutricional tomando el Z Score Peso/talla se observó que la desnutrición aguda moderada paso de 5,3\% (IC 2,4-11,4) a 4,4\% (IC 1,8$10,3)$, el peso adecuado para la talla tuvo un aumento de $78,8 \%$ (IC 70,1-85,4) a 84,9\% (76,9-90,5), donde también se observaron reducciones de las prevalencias en los indicadores de riesgo de sobrepeso, sobrepeso y obesidad (tabla 2).

$\mathrm{Al}$ analizar el cambio en los indicadores antropométricos según el estado nutricional inicial se observó que hubo un aumento de las medias de puntuaciones Z IMC/edad y Z Peso/talla de aproximadamente 0,4 puntos en el subgrupo de diagnóstico inicial de bajo peso, siendo estadísticamente significativos $(p=0,0)$, aunque esto no se vio reflejado en la puntuación $\mathrm{Z}$ Talla/edad (tabla 3).

La diferencia de la puntuación de $\mathrm{Z}$ de IMC/edad fue de 0,2 en el grupo mayor a dos años mientras que fue de $-0,5$ en los menores a esa edad $(p=0,0)($ tabla 4$)$.

En cuando a los cambios en la media de Z Talla/ edad se constató diferencias significativas según sexo, siendo $-0,1$ puntos en niñas y 0,3 en niños $(p=0,0)$. En menores de dos años fue de 0,5 y en mayores fue $-0,1$ $(\mathrm{p}=0,0)($ tabla 4$)$.

En la media de Z Peso/talla la puntuación aumentó de manera estadísticamente significativa en el subgrupo mayor de dos años con respecto al de edad menor o igual a dos años $(p=0,0)$. En este indicador se encontró un coeficiente de 0,0 en el grupo que recibió programa de trasferencia directa mientras que el coeficiente fue de $-0,3$ en el que no lo recibió, siendo diferencias estadísticamente significativas $(\mathrm{p}=0,1)$ (tabla 4$)$.

El 97\% de niños/as mantuvo su estado nutricional adecuado o superior desde el inicio al final del programa, mientras que solo el 3\% con bajo peso o riesgo de bajo peso tuvo mejoras del diagnóstico medido por el Z Score de IMC/edad pasando a una categoría superior.

Un 56,1\% de niños/as con diagnóstico inicial de baja talla o riesgo de baja talla pasaron a tener una talla adecuada a su edad ( $Z$ Talla/edad) y según el $Z$ Score Peso/talla un $33 \%$ que presentaba algún tipo de desnutrición o en riesgo mejoró su diagnóstico inicial. Sin embargo en todos los análisis estas diferencias no fueron estadísticamente significativas (tabla 5). 


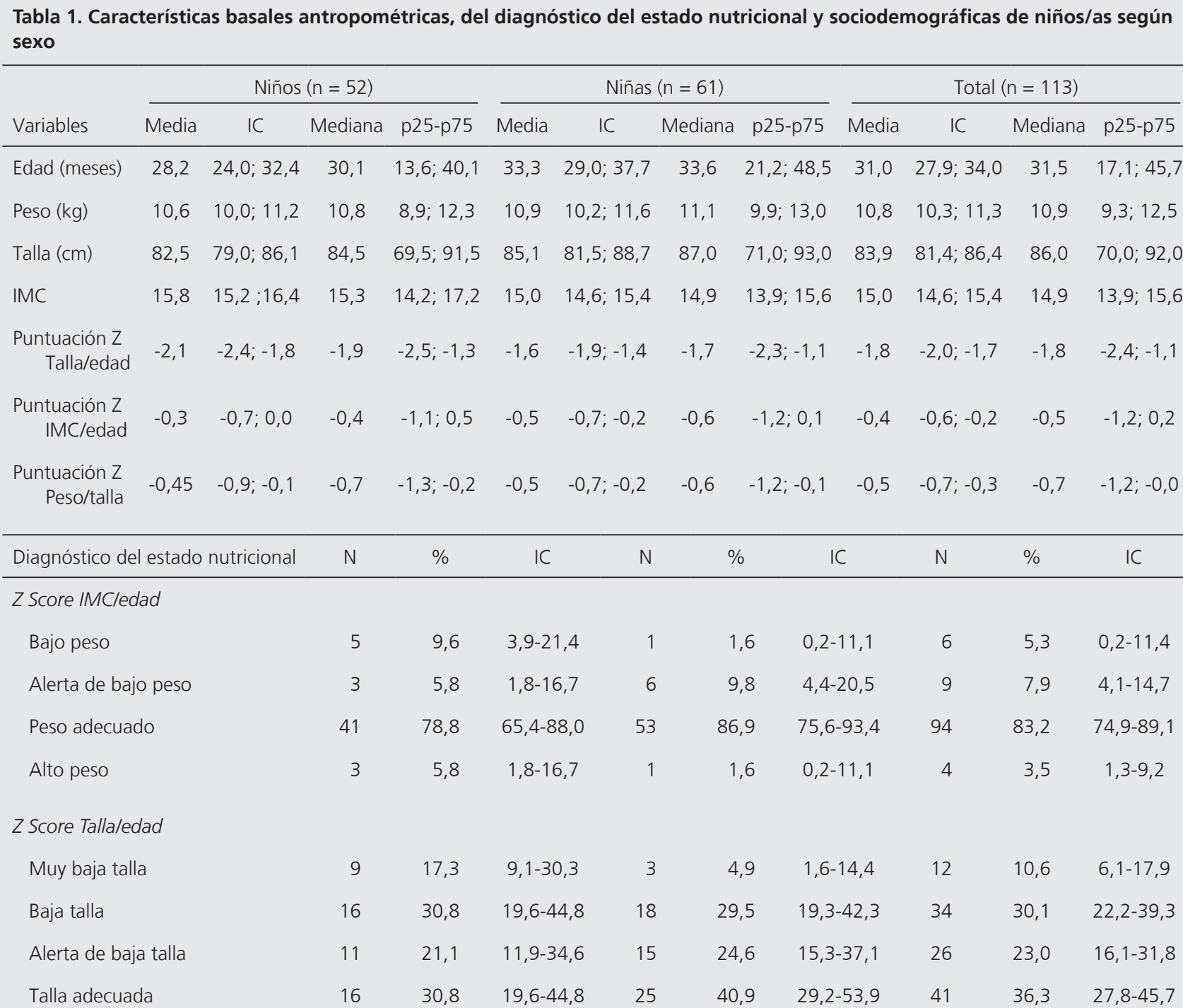

\section{Z Score Peso/talla}

\begin{tabular}{|c|c|c|c|c|c|c|c|c|c|}
\hline Desnutrición aguda severa & 1 & 1,9 & $0,3-12,9$ & 0 & 0 & 0 & 1 & 0,9 & $0,1-6,2$ \\
\hline Desnutrición aguda moderada & 5 & 9,6 & $3,9-21,4$ & 1 & 1,6 & $0,2-11,1$ & 6 & 5,3 & $2,4-11,4$ \\
\hline Riesgo de desnutrición aguda & 3 & 5,8 & $1,8-16,7$ & 8 & 13,1 & $6,6-24,4$ & 11 & 9,7 & $5,4-16,9$ \\
\hline Peso adecuado para la talla & 40 & 76,9 & $63,3-86,6$ & 49 & 80,3 & $68,2-88,6$ & 89 & 78,8 & $70,1-85,4$ \\
\hline Riesgo de sobrepeso o sobrepeso & 1 & 1,9 & $0,3-12,9$ & 3 & 4,9 & $1,6-14,4$ & 4 & 3,5 & $1,3-9,2$ \\
\hline Obesidad & 2 & 3,8 & $0,9-14,5$ & 0 & 0 & 0 & 2 & 1,8 & $0,4-6,9$ \\
\hline Recibe protección social & $\mathrm{N}$ & $\%$ & IC & $\mathrm{N}$ & $\%$ & IC & $\mathrm{N}$ & $\%$ & IC \\
\hline Asistencia alimentaria & 20 & 38,5 & $26,1-52,5$ & 18 & 29,5 & $19,3-42,3$ & 38 & 33,6 & $25,4-42,9$ \\
\hline Transferencia directa & 32 & 61,5 & $47,5-73,9$ & 38 & 62,3 & $49,4-73,7$ & 70 & 61,9 & $52,5-70,5$ \\
\hline
\end{tabular}

Edad se define como años decimales cumplidos al momento del relevamiento de datos. Peso coporal expresado en $\mathrm{Kg}$. Talla expresada en $\mathrm{cm}$. IMC: Indice de masa corporal. Puntuación Z se define como: líneas de referencia de las curvas de crecimiento, también conocidas como puntuación de desviación estándar (DE) según los lineamientos de la OMS. Recibe proteccion social se define como: estar recibiendo el Programa Diconsa y/o Programa Oprotunidades al momento de ingresar a la intervención. IC: Intervalo de confianza al 95\%. p25-p75: Percentilos 25 y 75. 
Tabla 2. Comparación de las variables antropométricas y del diagnóstico del estado nutricional de niños/as al incio y final del programa $(n=113)$

\begin{tabular}{|c|c|c|c|c|}
\hline \multirow[b]{2}{*}{ Variables nutricionales } & \multicolumn{2}{|c|}{ Inicio } & \multicolumn{2}{|c|}{ Final } \\
\hline & Media (IC) & Mediana (p25-p75) & Media (IC) & Mediana (p25-p75) \\
\hline Puntuación Z Talla/edad & $-1,8(-2,0 ; 1,7)$ & $-1,8(-2,4 ;-1,1)$ & $-1,9(-2,1 ;-1,1)$ & $-1,8(-2,4 ;-1,3)$ \\
\hline Puntuación Z IMC/edad & $-0,4(-0,6 ;-0,2)$ & $-0,5(-1,2 ;-0,2)$ & $-0,4(-0,6 ;-0,3)$ & $-0,5(-1,0 ; 0,1)$ \\
\hline Puntuación Z Peso/talla & $-0,5(-0,7 ;-0,3)$ & $-0,7(-1,2 ;-0,0)$ & $-0,6(-0,8 ;-0,4)$ & $-0,6(-1,1 ;-0,1)$ \\
\hline Diagnóstico del estado nutricional & $n / N$ & $\%(I C)$ & $\mathrm{n} / \mathrm{N}$ & $\%(I C)$ \\
\hline \multicolumn{5}{|l|}{ Según Z Score IMCledad } \\
\hline Bajo peso & $6 / 113$ & $5,3(2,4 ; 11,4)$ & $5 / 113$ & $4,4(1,8 ; 10,3)$ \\
\hline Alerta de bajo peso & 9/113 & $7,9(4,1 ; 14,7)$ & $8 / 113$ & $7,1(3,5 ; 13,6)$ \\
\hline Peso adecuado & $94 / 113$ & $83,2(75,9 ; 89,1)$ & $98 / 113$ & $86,7(79,0 ; 91,9)$ \\
\hline Alto peso & $4 / 113$ & $3,5(1,3 ; 9,2)$ & $1 / 113$ & $0,9(0,1 ; 0,6)$ \\
\hline Muy alto peso & NA & NA & $1 / 113$ & $0,9(0,1 ; 0,6)$ \\
\hline \multicolumn{5}{|l|}{ Según Z Score Tallaledad } \\
\hline Muy baja talla & $12 / 113$ & $10,6(6,1 ; 17,9)$ & $7 / 113$ & $6,2(2,9 ; 12,6)$ \\
\hline Baja talla & $34 / 113$ & $30,1(22,2 ; 39,3)$ & $34 / 113$ & $30,1(22,2 ; 39,3)$ \\
\hline Alerta de baja talla & 26/113 & $23,0(16,1 ; 31,8)$ & $31 / 113$ & $27,4(19,9 ; 36,5)$ \\
\hline Talla adecuada & $41 / 113$ & $36,3(27,8 ; 45,7)$ & $40 / 113$ & $35,4(27,0 ; 44,8)$ \\
\hline Muy alta talla & NA & NA & $1 / 113$ & $0,9(0,1 ; 0,6,2)$ \\
\hline \multicolumn{5}{|l|}{ Según Z Score Peso/talla } \\
\hline Desnutrición aguda severa & $1 / 113$ & $0,9(0,1 ; 6,2)$ & $1 / 113$ & $0,9(0,1 ; 6,2)$ \\
\hline Desnutrición aguda moderada & $6 / 113$ & $5,3(2,4 ; 11,4)$ & $5 / 113$ & $4,4(1,8 ; 10,3)$ \\
\hline Riesgo de desnutrición aguda & $11 / 113$ & $9,7(5,4 ; 16,9)$ & $8 / 113$ & $7,1(3,5 ; 13,6)$ \\
\hline Peso adecuado para la talla & $89 / 113$ & $78,8(70,1 ; 85,4)$ & $96 / 113$ & $84,9(76,9 ; 90,5)$ \\
\hline Riesgo de sobrepeso o sobrepeso & $4 / 113$ & $3,5(1,3 ; 9,2)$ & $2 / 113$ & $1,8(0,4 ; 6,9)$ \\
\hline Obesidad & $2 / 113$ & $1,8(0,4 ; 6,9)$ & $1 / 113$ & $0,9(0,1 ; 6,2)$ \\
\hline
\end{tabular}

IC: Intervalo de confianza al 95\%. p25-p75: Percentilos 25 y 75. NA: no aplica.

Tabla 3. Cambio de los indicadores antropométricos de niños/as entre el inicio y final del programa según el diagnóstico nutricional inicial $(\mathrm{N}=112)$

\begin{tabular}{|c|c|c|c|c|c|c|c|c|c|c|c|c|}
\hline \multirow{3}{*}{$\begin{array}{l}\text { Diagnóstico inicial } \\
\text { Cambio en los indicadores } \\
\text { antropométricos }\end{array}$} & \multicolumn{12}{|c|}{ Z Score IMC/edad } \\
\hline & \multicolumn{3}{|c|}{ Bajo peso } & \multicolumn{3}{|c|}{ Riesgo de bajo peso } & \multicolumn{3}{|c|}{ Peso adecuado } & \multicolumn{3}{|c|}{ Peso alto } \\
\hline & Coef. & IC & $p$ & Coef. & IC & $p$ & Coef. & IC & $p$ & Coef. & IC & $\mathrm{p}$ \\
\hline Puntuación Z Talla/edad & $-0,3$ & $-0,6 ; 0,1$ & 0,1 & $-0,2$ & $-0,3 ;-0,0$ & 0,0 & 0,1 & $-0,1 ; 0,3$ & 0,3 & 0,6 & $-1,1 ; 2,3$ & 0,3 \\
\hline Puntuación Z IMC/edad & 0,5 & 0,$2 ; 0,7$ & 0,0 & 0,3 & 0,$1 ; 0,6$ & 0,1 & $-0,1$ & $-0,2 ; 0,1$ & 0,2 & $-0,8$ & $-2,4 ; 0,8$ & 0,2 \\
\hline Puntuación Z Peso/talla & 0,4 & 0,$2 ;-0,6$ & 0,0 & 0,3 & 0,$5 ; 0,6$ & 0,0 & $-0,1$ & $-0,3 ; 0,0$ & 0,1 & $-1,4$ & $-3,6 ; 0,7$ & 0,1 \\
\hline \multicolumn{13}{|c|}{$\begin{array}{l}\text { Coef.: coeficiente obtenido por un modelo de regresión lineal simple (bivariado), donde las variables dependientes fueron: diferencia en la } \\
\text { puntuación Z de IMC/edad, Talla/edad (solo en niños/as con desnutrición aguda en la etapa basal) y Peso/talla entre la etapa inicial y a los } \\
4 \text { meses de seguimiento. La variable independiente fue el diagnótico inicial obtenido por el Z Score IMC/edad. p: nivel de significancia esta- } \\
\text { dística }<0,05 \text {. IC: intervalo de confianza al } 95 \% \text {. Puntuación Z: líneas de referencia de las curvas de crecimiento también conocidas como } \\
\text { puntuación de desviación estándar (DE) según los lineamientos de la OMS. }\end{array}$} \\
\hline
\end{tabular}


Tabla 4. Diferencia media de los indicadores antropométricos en niños/as entre el inicio y final del programa según variables sociodemográficas $(n=112)$

\begin{tabular}{|c|c|c|c|c|c|c|c|c|c|c|c|c|c|}
\hline \multirow[b]{2}{*}{ Demográficas } & \multicolumn{5}{|c|}{ Z IMC/edad } & \multicolumn{4}{|c|}{ Z Talla/edad } & \multicolumn{4}{|c|}{ Z Peso/talla } \\
\hline & $\mathrm{N}$ & Media* & $\mathrm{EE}$ & IC & $\mathrm{p}$ & Media* & EE & IC & $p$ & Media* & EE & IC & $\mathrm{p}$ \\
\hline \multicolumn{14}{|l|}{ Sexo } \\
\hline Niñas & 60 & 0,0 & 0,1 & $-0,2 ; 0,2$ & & $-0,1$ & 0,1 & $-0,3 ; 0,1$ & & $-0,0$ & 0,1 & $-0,3 ; 0,1$ & \\
\hline Niños & 52 & $-0,1$ & 0,1 & $-0,3 ; 0 ; 1$ & 0,3 & 0,3 & 0,1 & 0,$0 ; 0,5$ & 0,0 & $-0,2$ & 0,1 & $-0,4 ; 0,0$ & 0,4 \\
\hline \multicolumn{14}{|l|}{ Edad } \\
\hline Menor o igual a 2 años & 39 & $-0,5$ & 0,2 & $-0,8 ;-0,2$ & & 0,5 & 0,2 & 0,$0 ; 0,9$ & & $-0,7$ & 0,2 & $-1,0 ; 0,3$ & \\
\hline Mayor a 2 años & 73 & 0,2 & 0,0 & 0,$1 ; 0,3$ & 0,0 & $-0,1$ & 0,0 & $-0,2 ;-0,1$ & 0,0 & 0,2 & 0,0 & 0,$1 ; 0,3$ & 0,0 \\
\hline \multicolumn{14}{|l|}{ Recibe trasferencia directa } \\
\hline No & 42 & $-0,2$ & 0,1 & $-0,5 ; 0,1$ & & 0,1 & 0,2 & $-0,2 ; 0,5$ & & $-0,3$ & 0,2 & $-0,7 ; 0,0$ & \\
\hline Si & 70 & 0,0 & 0,1 & $-0,1 ; 0,2$ & 0,1 & 0,0 & 0,1 & $-0,1 ; 0,2$ & 0,4 & 0,0 & 0,1 & $-0,1 ; 0,1$ & 0,0 \\
\hline \multicolumn{14}{|c|}{ Recibe asistencia alimentaria } \\
\hline No & 74 & $-0,1$ & 0,1 & $-0,3 ; 0,1$ & & 0,1 & 0,1 & $-0,1 ; 0,3$ & & $-0,1$ & 0,1 & $-0,4 ; 0,0$ & \\
\hline Si & 38 & $-0,0$ & 0,1 & $-0,2 ; 0,2$ & 0,6 & 0,1 & 0,1 & $-0,2 ; 0,3$ & 1,0 & $-0,0$ & 0,1 & $-02 ; 0,2$ & 0,4 \\
\hline
\end{tabular}

*Diferencia media en la puntuación Z de IMC/edad, Talla/edad y Peso/talla entre la etapa inicial y a los 4 meses de seguimiento. p: nivel de significancia estadística < 0,05. IC: intervalo de confianza al 95\%. EE: error estándar.

Tabla 5. Mantenimiento la categoría diagnóstica del estado nutricional de niños/as entre el inicio y final del programa $(n=113)$

\begin{tabular}{|c|c|c|c|c|}
\hline \multirow{3}{*}{$\begin{array}{l}\text { Diagnóstico final } \\
\text { Diagnóstico Inicial }\end{array}$} & \multicolumn{4}{|c|}{ Z Score IMC/edad } \\
\hline & Bajo peso y riesgo de bajo peso & Peso adecuado o más & Total & \multirow[t]{2}{*}{$\mathrm{p}$} \\
\hline & n $\quad(\%)$ & n $\quad(\%)$ & n $\quad(\%)$ & \\
\hline Bajo peso y riesgo de bajo peso & $10(100)$ & $0(0)$ & $10(100)$ & \\
\hline \multirow[t]{2}{*}{ Peso adecuado o superior } & $3(2,9)$ & $100(97,1)$ & $103(100)$ & 0,1 \\
\hline & \multicolumn{3}{|c|}{ Z Score Talla/edad } & \\
\hline Diagnóstico final & Baja talla y riesgo de baja talla & Talla adecuada o más & Total & \multirow[t]{2}{*}{$\mathrm{p}$} \\
\hline Diagnóstico Inicial & n $\quad(\%)$ & $n \quad(\%)$ & n $\quad(\%)$ & \\
\hline Baja talla y riesgo de baja talla & $61(43,9)$ & $11(56,1)$ & $72(100)$ & \\
\hline \multirow[t]{2}{*}{ Talla adecuada o superior } & $11(4,5)$ & $30(95,5)$ & $41(100)$ & 1,0 \\
\hline & \multicolumn{3}{|c|}{ Z Score Peso/talla } & \\
\hline \multirow{2}{*}{$\begin{array}{l}\text { Diagnóstico final } \\
\text { Diagnóstico Inicial }\end{array}$} & $\begin{array}{l}\text { Desnutrición aguda severa y } \\
\text { moderada y riesgo de desnutrición }\end{array}$ & $\begin{array}{l}\text { Peso adecuado } \\
\text { o superior }\end{array}$ & Total & \multirow[t]{2}{*}{$\mathrm{p}$} \\
\hline & n $\quad(\%)$ & n $\quad(\%)$ & n $\quad(\%)$ & \\
\hline \multirow{2}{*}{$\begin{array}{l}\text { Desnutrición aguda severa y moderada } \\
\text { y riesgo de desnutrición } \\
\text { Peso adecuado o superior }\end{array}$} & $12(66,7)$ & $6(33,3)$ & $18(100)$ & \multirow{2}{*}{0,2} \\
\hline & $2(2,1)$ & $93(97,9)$ & $95(100)$ & \\
\hline
\end{tabular}

p: nivel de significancia estadística $<0,05$.

\section{Discusión}

Se concluye que el programa comunitario llevada a cabo en niños y niñas menores de 5 años de una zona rural de Chiapas, México durante los cuatro meses de su implementación ha contribuido a mejorar los indicadores antropométricos de puntuación Z IMC/ edad y de Peso/talla, principalmente en los grupos con diagnóstico inicial de bajo peso. Sin embargo, no se encontraron efectos aparentes en indicadores relacionados a la desnutrición crónica en la población estudiada. 
La malnutrición infantil en contextos rurales es un problema prioritario en la agenda pública de los países de América Latina y el Caribe de hace décadas. Los primeros estudios relevantes sobre el tema datan del año $1975^{25}$.

En la región, existen algunos antecedentes publicados sobre intervenciones enfocadas a mejorar la malnutrición infantil en menores de cinco años ${ }^{25,26-28}$; sin embargo, son escasas las publicaciones sobre intervenciones comunitarias con enfoques holísticos abordando tanto aspectos individuales como también socioambientales en donde se desenvuelven las biografías de niños y niñas insertos en el medio rural.

$\mathrm{Al}$ inicio de la intervención se observó una alta prevalencia de desnutrición crónica, siendo concordante con el estudio llevado a cabo en niños de México de Rivera y col. ${ }^{14}$.

Un resultado interesante de nuestro estudio fue el aumento del puntaje Z del IMC/edad y Peso/talla, principalmente en los subgrupos con bajo peso y riesgo de bajo peso entre el inicio y el final del programa siendo este resultado similar con lo publicado por otro estudio realizado en Chile ${ }^{29}$.

Casi una tercera parte de los niños estudiados que entraron a la intervención con bajo peso o riesgo de bajo peso, evolucionaron positivamente su diagnóstico de peso para la talla. Sin embargo, en un $4 \%$ de los que ingresaron con talla para la edad adecuada su estado nutricional empeoró. Esto podría explicarse por el corto periodo de intervención o bien por potenciales imprecisiones en las mediciones antropométricas, aunque este último aspecto pudo estar controlado a partir de la capacitación al personal de salud antes de comenzar la implementación del programa.

Si bien se observó que la prevalencia de muy baja talla se redujo levemente entre el inicio y final del programa, no se encontraron efectos del programa en indicadores relacionados a la desnutrición crónica (Z Talla/edad) en la población bajo cobertura. Somos conscientes que el corto periodo de tiempo de implementación del programa (4 meses) que invalida el poder alcanzar efectos en la talla. Como lo plantea Galván y col..$^{30}$ aún cuando existan ganancias de estatura, la reducción de las prevalencias de desnutrición crónica sucedería a largo plazo.

También se encontraron mejoras en la talla/edad en el subgrupo de menores de dos años. Esto podría relacionarse con el denominado periodo de "mil días de oportunidades para intervenciones nutricionales" ${ }^{\prime 1}$, donde existe una capacidad de crecimiento compensador después de un periodo de retraso en el crecimiento. Nuestros resultados son consistentes con otros estudios realizados en América Latina ${ }^{32-34}$.

En nuestro trabajo se evidenciaron mejoras en el subgrupo que recibió programa de protección social a través de transferencia directa en los indicadores de Z Peso/talla y Z Talla/edad (siendo estadísticamente significativo solo en el primero caso). Esto estaría indicando el potencial que tienen estos programas para contribuir a mejorar la nutrición infantil ${ }^{30}$.

En este estudio también se demostraron diferencias según sexo en la media de cambio de la puntuación $\mathrm{Z}$ talla/edad antes y después de la intervención, teniendo mejores resultados en el subgrupo de niños con respecto a las niñas. Es importante destacar que al inicio de la intervención los niños presentaron una prevalencia mayor de desnutrición crónica que las niñas, siendo un resultado concordante con otros estudios realizados en América Latina ${ }^{34,35}$. Posiblemente durante la intervención, en los niños podría haber existido un aceleramiento mayor del crecimiento y un catch-up rápido compensador como lo plantea Victora C. ${ }^{31}$.

Una de las principales fortalezas del estudio radica en la importancia de evaluar el impacto de programas comunitarios impulsados desde el tercer sector y el Estado. La evidencia indica que existen importantes inequidades socioeconómicas en la desnutrición tanto en áreas urbanas como rurales ${ }^{36}$ por lo que se necesita implementar y evaluar programas comunitarios locales orientados a mejorar la malnutrición y reducir estas brechas de inequidad.

El estudio presenta como principal limitación el corto periodo de intervención lo que invalida la posibilidad de ver cambios globales y mejoras concretas en el diagnóstico de baja talla e impactos en el crecimiento longitudinal en el grupo expuesto al programa.

Otra limitación radica en la ausencia de un grupo control. Sin embargo, es importante considerar que existe una disociación entre la evidencia obtenida en estudios experimentales en el campo de la salud y la aplicación en el mundo real ${ }^{37}$, donde este tipo de diseños flexibles se podría considerar aceptable y realista.

Este estudio aporta evidencia científica al campo de la investigación evaluativa de programas de nutrición comunitaria con enfoque integral destinadas a niños y niñas pertenecientes a zonas rurales, siendo un tema de escasos antecedentes documentados en la región.

\section{Conclusiones}

Si bien el programa de nutrición comunitaria tuvo impacto positivo en indicadores antropométricos vinculados a la desnutrición aguda en la primera infancia no se observaron cambios en los indicadores nutricionales relacionados a la desnutrición crónica, lo que podría deberse al corto periodo de tiempo de la intervención nutricional. 


\section{Responsabilidades Éticas}

Protección de personas y animales: Los autores declaran que los procedimientos seguidos se conformaron a las normas éticas del comité de experimentación humana responsable y de acuerdo con la Asociación Médica Mundial y la Declaración de Helsinki.

Confidencialidad de los datos: Los autores declaran que han seguido los protocolos de su centro de trabajo sobre la publicación de datos de pacientes.

Derecho a la privacidad y consentimiento informado: Los autores han obtenido el consentimiento informado de los pacientes y/o sujetos referidos en el artículo. Este documento obra en poder del autor de correspondencia.

\section{Conflicto de intereses}

Los autores declaran no tener conflicto de intereses.

\section{Agradecimientos}

A niños, niñas y sus familias pertenecientes a la comunidad de La Soledad del Estado de Chiapas que formaron parte del programa y al equipo de salud de Partners in Health filial México que trabajó en el territorio.

\section{Referencias}

1. Organización de las Naciones Unidas. Convención sobre los Derechos del Niño. ONU; 1989. Disponible en http:// www.un.org/es/events/childrenday/pdf/ derechos.pdf

2. Mieles MD Acosta A. Calidad de vida y derechos de la infancia: un desafío presente. Revista Latinoamericana de Ciencias Sociales, Niñez y Juventud. 2012;10(1):205-17.

3. Fondo de las Naciones Unidas para la infancia. La infancia. [Internet]. Unicef; 2017. Disponible en https://www.unicef. org/mexico/spanish/ninos.html

4. Organización Mundial de la Salud. Subsanar las desigualdades en una generación. Alcanzar la equidad sanitaria actuando sobre los determinantes sociales de la salud. Ginebra, Suiza: OMS; 2008. Disponible en http://apps.who.int/iris/ bitstream/handle/10665/69830/WHO_ IER_CSDH_08.1_spa.pdf;jsessionid= $=12$ E7 4A49D8719F2BAA9689EF80F7AF42?sequ ence $=1$.

5. Martorell R. Efectos de la desnutrición en la salud y desarrollo humano y estrategias efectivas para su prevención. Salud Pública Méx. 2007;49 (Ed Esp 1):151.

6. Fondo de las Naciones Unidas para la Infancia. Desnutrición infantil en América Latina y el Caribe. CEPAL, Unicef: Santiago, Chile; 2006. Disponible en http://www.oda-alc.org/ documentos/1367000434.pdf.

7. Breilh J. La epidemiología crítica: una nueva forma de mirar la salud en el espacio urbano. Salud Colectiva. 2010; 6(1):83-101.

8. Ortiz-Andrellucchi, L. Peña Quintana y col. Desnutrición infantil, salud y pobreza: intervención desde un programa integral. Argentina. Nutr Hosp. 2006; 21(4):533-41.

9. De Castro J. El Hambre problema universal. Buenos Aires: Editorial Leviatán; 1983.

10. Organización Mundial de la Salud. Malnutrición: OMS; 2017. Disponible en http://www.who.int/mediacentre/ factsheets/malnutrition/es/.

11. Secretaría de Salud de México. Encuesta Nacional de Salud y Nutrición de Medio Camino; 2016. Disponible en http:// transparencia.insp.mx/2017/auditoriasinsp/12701_Resultados_Encuesta_ ENSANUT_MC2016.pdf.

12. Fernández Cantón SB, Viguri Uribe R. La mortalidad por desnutrición en México en menores de cinco años, 1990-2009. Boletín médico del Hospital Infantil de México. 2010; 67:(5)471-3.

13. Ayala-Gaytán EA, Díaz Durán-Hernández A. Infraestructura, ingreso y desnutrición infantil en México. Salud Pública Méx. 2015; 57:22-8.

14. Rivera-Dommarco JÁ, Cuevas-Nasu L, González de Cosío T, Shamah-Levy T, García-Feregrino R. Desnutrición crónica en México en el último cuarto de siglo: análisis de cuatro encuestas nacionales. Salud Pública Méx. 2013; 55(2):S161-S9.

15. Kac G, García Alvear JL. Epidemiología de la desnutrición en Latinoamérica: situación actual. Nutr Hosp. 2010; 25(3):50-6

16. Organización de las Naciones Unidas para la Alimentación y la Agricultura. Organización Panamericana de la Salud. Panorama de la Seguridad Alimentaria y Nutricional en América Latina y el Caribe. Santiago de Chile: FAO/OPS; 2017. Disponible en http://www.fao.org/3/ai7914s.pdf.

17. Fondo de las Naciones Unidas para la Infancia. El estado mundial de la infancia
2016. Una oportunidad para cada niño. New York: Unicef; 2016. Disponible en https://www.unicef.org/spanish/ publications/files/UNICEF_SOWC_2016_ Spanish.pdf

18. Akombi BJ, Agho KE, Hall JJ, Wali N, Renzaho AMN, Merom D. Stunting, Wasting and Underweight in Sub-Saharan Africa: A Systematic Review. Int J Environ Res Public Health. 2017;14(8):863.

19. Maluccio JA, Hoddinott J, Behrman JR, Martorell R, Quisumbing AR, Stein AD. The impact of improving nutrition during early childhood on education among Guatemalan adults. The Economic Journal. 2009;119(537):734-63.

20. Rodríguez-Martín A, Novalbos-Ruiz JP, Jiménez-Rodríguez A, Baglietto Ramos M, Romero Sánchez JM. Implicaciones de la desnutrición en atención primaria. Nutr Hosp. 2010; 25(3):67-79.

21. Jiménez-Benítez D, Rodríguez-Martín A, Jiménez-Rodríguez R. Análisis de determinantes sociales de la desnutrición en Latinoamérica. Nutr Hosp. 2010; 25(3):18-25.

22. Compañeros en Salud. Nuestro impacto; 2017. Disponible en https:// companerosensalud.mx/impacto/

23. Word Health Organitation. Child Growth Standards: Methods and development. Geneva: WHO; 2006. Available in http:// www.who.int/childgrowth/standards/ Technical_report.pdf

24. Bronfenbrenner U. The Ecology of Human Development. Cambridge: Harvard University Press; 1979.

25. Instituto de Nutrición de Centroamérica y Panamá. Nutricion, crecimiento y desarrollo. INCAP; 1975. Disponible en http://iris.paho.org/xmlui/bitstream/ handle/123456789/17627/v78n1p38. pdf?sequence $=1$

26. Bonilla Catherine, Híjar Gisely, Márquez 
Delia, Aramburú Adolfo, Aparco Juan Pablo, Gutiérrez Ericson L. Intervenciones para prevenir la aparición de sobrepeso y obesidad en niños menores de cinco años. Rev. Perú. Med. Exp. Salud Publica. 2017; 34(4):682-9.

27. Rodríguez O Lorena, Pizarro Q Tito, Benavides M Xenia, Atalah S Eduardo. Evaluación del impacto de una intervención alimentario nutricional en niños chilenos con malnutrición por déficit. Rev. Chil. pediatr. 2007; 78(4):376-83.

28. Vitolo MR, Bortolini GA, Campagnolo PD, Hoffman DJ. Maternal dietary counseling reduces consumption of energy-dense foods among infants: a randomized controlled trial. J Nutr Educ Behav. 2012; 44(2):140-7.

29. Ratner RG, Durán SA, Garrido MJL, Balmaceda SH., Jadue LH, Atalah ES. Impacto de una intervención en alimentación y actividad física sobre la prevalencia de obesidad en escolares. Nutr. Hosp. 2013; 28(5):1508-14.

30. Galván M, Amigo H. Programas destinados a disminuir la desnutrición crónica. Una revisión en América Latina. ALAN. 2007; 57(4):316-26.

31. Victora C. Los mil días de oportunidad para intervenciones nutricionales. De la concepción a los dos años de vida. Arch Argent Pediatr 2012; 110(4):311-7.

32. Buitrón D, Hurtig A, San Sebastián M. Estado Nutricional en niños naporunas menores de cinco años en la Amazonia ecuatoriana. Rev. Panam Salud Pública. 2004; 15(3):151-9.

33. Calvo E, et al. Estudios sobre Nutrición en menores de 3 años (Alimentación y Riesgo de desnutrición infantil). Estudio Colaborativo Multicéntrico. Ministerio de Salud y Ambiente de la Nación, Comisión Nacional de Programas de Investigación
Sanitaria CONAPRIS; 2003. Disponible en http://www.fmed.uba.ar/depto/ edunutri/Calvo_desnutricion_infantil.pdf

34. Sobrino M, Gutiérrez C, Cunha AJ, Dávila M, Alarcón J. Desnutrición infantil en menores de cinco años en Perú: tendencias y factores determinantes. Rev Panam Salud Publica. 2014;35(2):104-12.

35. Lafuente YKV, Rodriguez S, Fontaine V, Yañez VR. Prevalencia de la desnutrición crónica en niños menores de 5 años atendidos en el Centro de Salud Tacopaya, primer semestre gestión 2014. Gac Med Bol. 2016;39(1):26-29.

36. Fotso. JC.Child health inequities in developing countries: differences across urban and rural areas. Int J Equity Health. 2006;5:(9).

37. Gagliardino JJ, Costa Gil JE, Faingold MC, Litwak L, Funte GV. Insulina y control de la diabetes en la argentina. medicina (Buenos Aires). 2013; 73: 520-8. 